\title{
Antiplatelet and anticoagulant nonuse for acute myocardial infarction in the emergency department: An analysis from the National Hospital Ambulatory Medical Care Survey, 2002-2010
}

\author{
Ankur Sethi MBBS FACP, Rohit R Arora MD FACC FAHA, Sandeep Khosla MD FACC
}

\begin{abstract}
A Sethi, RR Arora, S Khosla. Antiplatelet and anticoagulant nonuse for acute myocardial infarction in the emergency department: An analysis from the National Hospital Ambulatory Medical Care Survey, 2002-2010. Curr Res Cardiol 2015;2(2):77-83.
\end{abstract}

BACKGROUND: Recent reports from quality improvement registries revealed high antiplatelet and anticoagulant use within $24 \mathrm{~h}$ of hospitalization for acute myocardial infarction (AMI).

OBJECTIVE: To produce an unbiased national estimate of the use of antiplatelets and anticoagulants during visits to the emergency department (ED) for AMI.

METHODS: Data from the National Hospital Ambulatory Medical Care Survey for AMI visits from 2002 to 2010 were analyzed. The complex survey design was taken into consideration to ensure that reliable national estimates were produced.

RESULTS: During the study period, there were 3,825,905 $\pm 308,534$ estimated eligible ED visits for AMI. Acetylsalicylic acid was not used in $55.5 \pm 2.9 \%, 55.7 \pm 4.0 \%$ and $55.2 \pm 4.8 \%$ of visits in 2002 to 2004,2005 to 2007 and 2008 to 2010 , respectively $\left(P_{\text {trend }}=0.96\right)$. The nonuse of thienopyridine or glycoprotein IIb/IIla inhibitors (GPI) decreased during this period $\left(92.9 \pm 1.4 \%, 88.1 \pm 2.6 \%\right.$ and $83.4 \pm 3.4 \%$, respectively; $\left.\mathrm{P}_{\text {trend }}=0.001\right)$. Anticoagulant nonuse did not change $(72.1 \pm 2.6 \%, 68.0 \pm 3.7 \%$ and $65.5 \pm 4.4 \%$, respectively; $\left.P_{\text {trend }}=0.14\right)$. Even in definitive AMI visits, nonuse was high (acetylsalicylic acid, $48.7 \pm 4.5 \%, 44.7 \pm 6.2 \%$ and $55.6 \pm 5.6 \%$, respectively, $\mathrm{P}_{\text {trend }}=0.31$; thienopyridine/GPI, $81.9 \% \pm 3.4 \%, 77.0 \pm 5.0 \%$ and $71.1 \pm 5.5 \%$, respectively, $\mathrm{P}_{\text {trend }}=0.001$; and anticoagulants, $49.3 \pm 9.6 \%$, $47.1 \pm 7.1 \%$ and $45.3 \pm 6.20 \%$, respectively, $\left.P_{\text {trend }}=0.24\right)$. ED visits in a metropolitan statistical area were more likely to receive acetylsalicylic acid (OR 2.27 [95\% CI 1.02 to 5.09]) and anticoagulants (OR 3.34 [95\% CI 1.54 to 7.24]). Similarly, visits evaluated by a consulting physician were more likely to receive thienopyridine/GPI (OR 2.24 [95\% CI 1.22 to 4.13]) and anticoagulants (OR 1.73 [95\% CI 1.14 to 2.63]).

CONCLUSION: In a significant proportion of AMI visits, including definitive AMI, antiplatelet and anticoagulant therapies were not administered in the ED.

Key Words: Emergency department; Quality of care; Acetylsalicylic acid; Myocardial infarction

visits to hospital outpatient departments and EDs conducted by the CDC and the National Center for Health Statistics (NCHS). Details regarding the survey design and multistage sampling are presented elsewhere $(7,8)$. Survey data from ED visits during 2002 to 2010 were used. During the study period, approximately $90.5 \%$ of invited in-scope hospitals with eligible EDs, defined as staffed $24 \mathrm{~h} /$ day, participated in the survey annually. Of these participating EDs, on average, $93.5 \%$ of the emergency service area responded completely or adequately by providing forms for at least one-half of the expected visits during the reported period. Therefore, the overall two-stage sampling unweighted response rate was $84.6 \%$. The visits of interest were selected based on the diagnosis entered on the patient record form. The NHAMCS survey instrument records one primary diagnosis and two other diagnoses related to the visit. The diagnosis may be tentative, provisional or definitive based on the physician's best judgment at that time. Two additional diagnoses may be recorded if related to the visit, which may include chronic conditions such as hypertension and depression. These diagnoses are coded in the dataset using International Classification of Diseases, Ninth Revision (ICD-9) codes. ICD-9 codes 410.X1 or 410.XO were used to identify the AMI visits. Visits by patients $\leq 18$ years of age or pregnant patients were excluded, as were visits with the following diagnoses based on ICD codes: gastrointestinal bleeding (578.X), intracranial bleed (430-, 431-, 432.X), coagulation defects (286.X), chronic liver disease (571.X), thrombocytopenia/purpura (287.XX) and gross hematuria (599.7X). In addition, as recommended by the AHA/ACC AMI performance measure document (5), visits in which the patient was

Study design and setting

\section{METHODS}

A secondary analysis of the ED data collected by the NHAMCS was performed. The NHAMCS is a national probability sample survey of

Division of Cardiology, Rosalind Franklin University of Medicine and Science, North Chicago, Illinois, USA

Correspondence: Dr Ankur Sethi, 3333 Green Bay Road, North Chicago, Illinois 60064, USA. Telephone 773-257-6452, fax 773-257-6726, e-mail drankursethi@gmail.com 


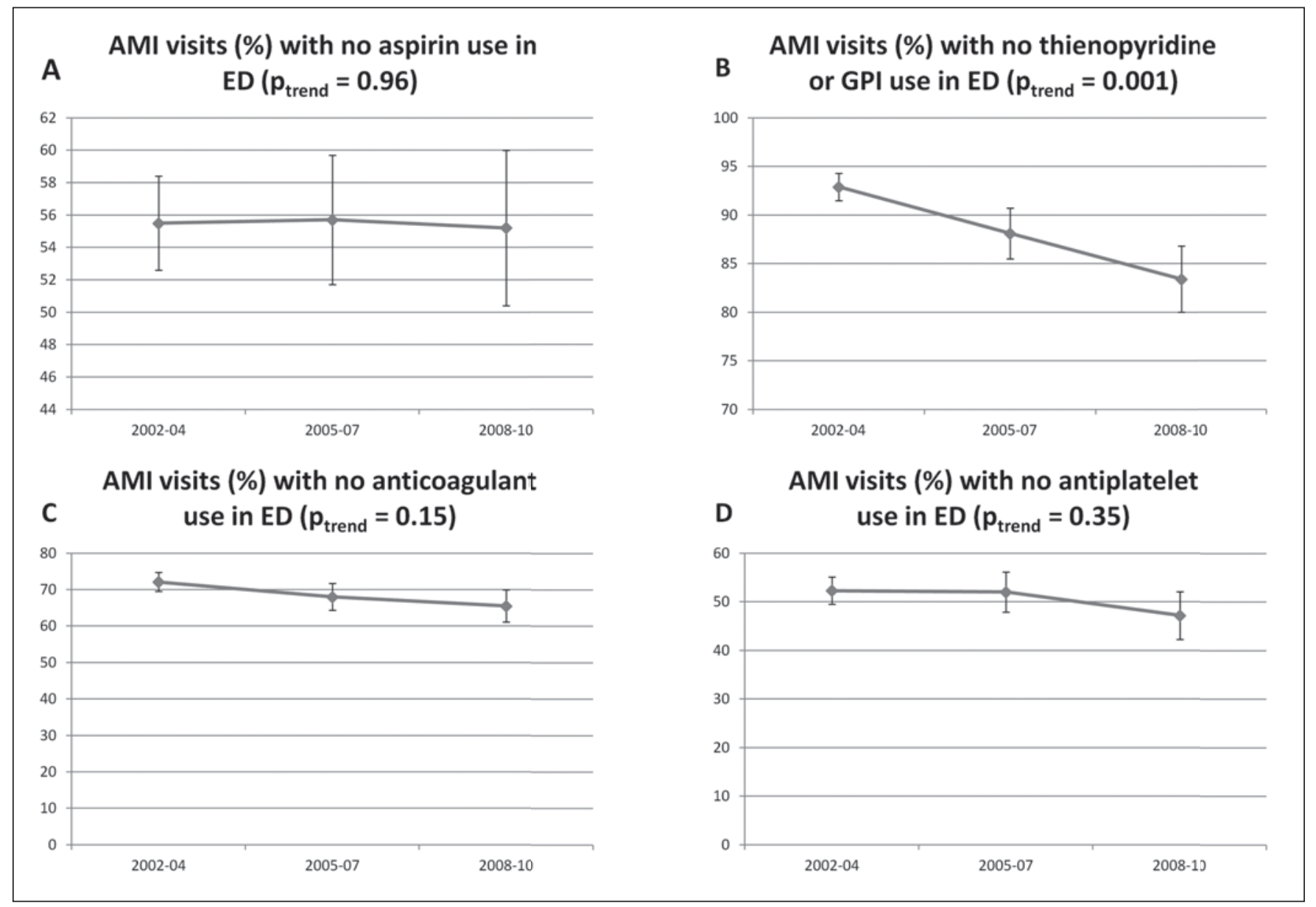

Figure 1) Proportion (\%) of all acute myocardial infarction (AMI) visits that did not receive A acetylsalicylic acid (aspirin), B thienopyridine or glycoprotein IIb/IIIa (GPI), C anticoagulants or D any antiplatelet agent in the emergency department (ED). The vertical bar represents standard error of the estimate

transferred to a different hospital, not admitted inpatient or observation status, left against medical advice, died in the ED or were dead on arrival were excluded, as were visits during which oral anticoagulants were prescribed or provided.

The following medications were identified to be administered in the ED, using generic drug codes 'gen' and 'drugid' for years 2002 to 2005 and 2006 to 2010, respectively: acetylsalicylic acid, clopidogrel, ticlopidine, tirofiban, abciximab, eptifibatide, heparin, dalteparin, fondaparinux, bivalirudin, enoxaparin, warfarin and dicumarol. The patient record form for all included years allowed recording of up to eight medications, except for 2002, when up to six medications were recorded.

The following predictor variables were used: age in years, sex, systolic blood pressure, pulse rate, length of the ED visit, arrival by an ambulance, race (black or white), ED location in a metropolitan statistical area (MSA), geographical location, evaluation by an ED attending physician and evaluation by another physician/on call fellow/consultant (consultant). A primary complaint of chest pain or related symptoms (reason for visit - 1050.0, 1050.1, 1050.2 and 1050.3), heart pain (reason for visit - 1265.0) and angina pectoris (reason for visit - 2515.0) were defined as typical symptoms of AMI. Visits with a primary diagnosis of AMI (prdiag1) not considered to be 'tentative' or 'rule out' were defined as definitive AMI.

The NHAMCS is approved annually by the Ethics Review Board of the NCHS with waivers of the requirements to obtain informed consent of patients and patient authorization for release of patient medical record data by health care providers.

\section{Outcomes}

The trends of nonuse of acetylsalicylic acid, thienopyridine or glycoprotein IIB/IIIA (GPI), and anticoagulants over the study period during ED visits for AMI were the primary outcomes. The predictors of use of these therapies were the secondary outcomes.

\section{Statistical analysis}

The analysis was performed using public-use data files. To account for the complex multistage survey design, a complex sample analysis was performed using stratification and clustering variables (Strata = CSTRATM, Cluster = CPSUM). Sample data were inflated with the patient visit weight 'PATWT' to produce national estimates. Threeyear intervals (2002 to 2004,2005 to 2007 , and 2008 to 2010) were used to attain a relative $\mathrm{SE}$ of $<30 \%$ and $>30$ records for all groups and subgroups, as recommended by the NCHS. The trends of continuous and categorical variables across the years were examined using complex sample general linear models and logistic models, respectively. The trends of nonuse of acetylsalicylic acid, thienopyridine or GPI, and anticoagulant were examined for all AMI visits and definitive AMI visits, as defined previously, by complex sample logistic regression with year interval as a predictor variable. The trend of acetylsalicylic acid nonuse was also examined in a subpopulation of visits not brought by ambulance. To evaluate the effect of the abovementioned predictor variables on the use of antiplatelet and anticoagulant, each predictor was entered in a univariate logistic model. The predictors with $\mathrm{P}<0.10$ were then entered in the final multivariable logistic model. $\mathrm{P}<0.05$ was considered to be statistically significant. The Bonferroni method was used to control for the type I error $(<0.05)$ due 


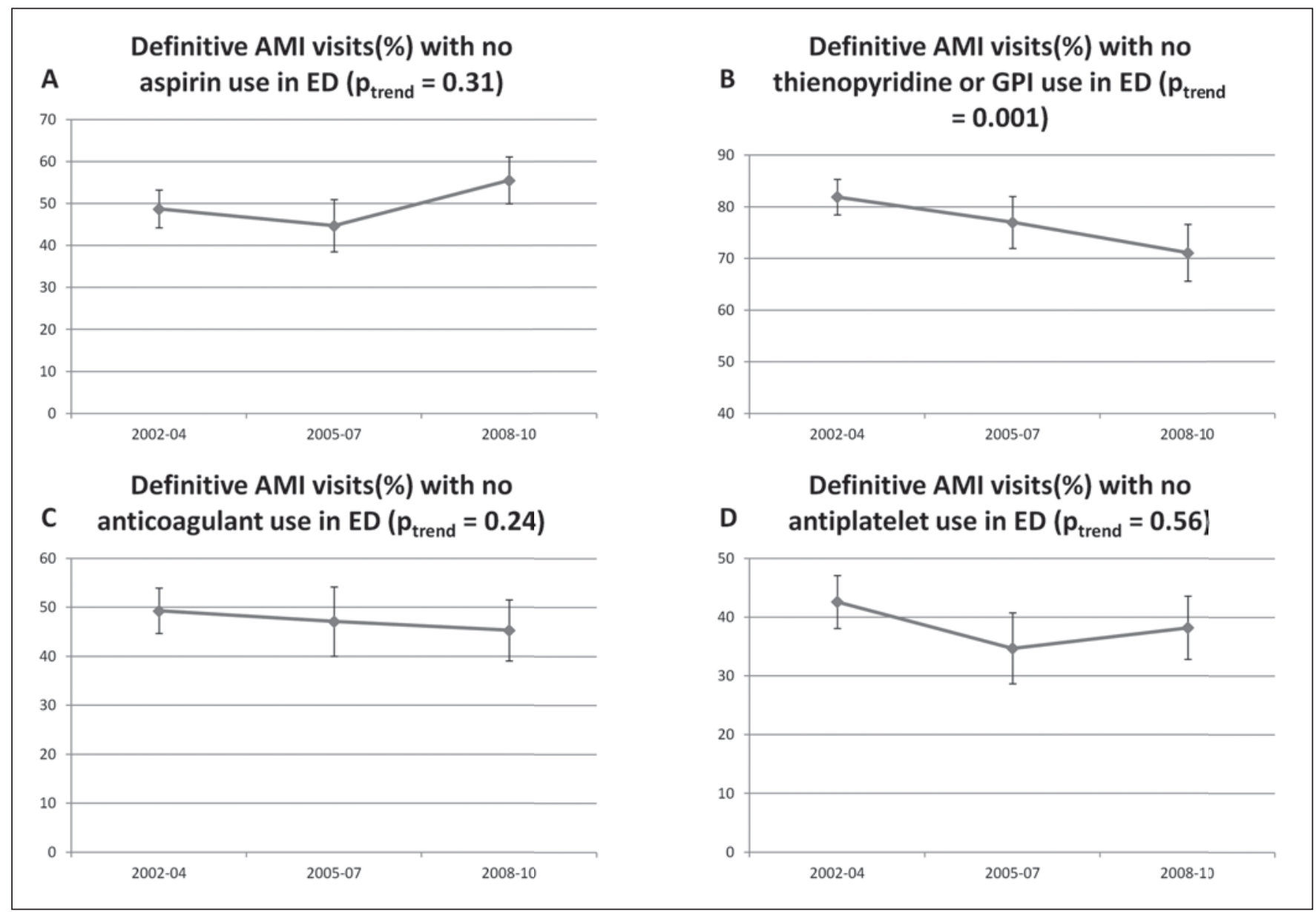

Figure 2) Proportion (\%) of definitive acute myocardial infarction (AMI) visits that did not receive A acetylsalicylic acid (asipirin), B thienopyridine or glycoprotein IIb/IIIa (GPI), C anticoagulants or D any antiplatelet agent in the emergency department (ED). The vertical bar represents standard error of the estimate

to multiple testing in multivariable models. All analysis was performed using SPSS version 17 (IBM Corporation, USA).

\section{RESULTS}

The estimated number of ED visits with a diagnosis of AMI during the nine-year period was 4,979,907 $\pm 357,445$ (Table 1). After exclusion of visits based on the criteria mentioned previously, 3,825,905 $\pm 308,534$ visits were considered to be eligible and were included in the denominator. The mean patient age varied from 64.01 to 66.0 years. The mean ( \pm SE) length of visit, systolic blood pressure and pulse are presented in Table 1. The statistically significant differences noted in these variables over the years were of minor clinical value. Slightly less than one-half of the AMI visits to the ED during the study period were female. The proportion of definitive AMI visits increased during the study period.

Acetylsalicylic acid was not administered in the ED in $55 \pm 2.9 \%$, $55.7 \pm 4.0 \%$ and $55.2 \pm 4.8 \%$ of AMI visits during 2002 to 2004,2005 to 2007 and 2008 to $2010\left(\mathrm{P}_{\text {trend }}=0.96\right)$, as shown in Figure $1 \mathrm{~A}$. Thienopyridine/GPI were not administered at $92.9 \pm 1.4 \%$ of visits in 2002 to 2004; however, their nonuse progressively decreased during the study period, and they were not administered at $88.1 \pm 2.6 \%$ and $83.4 \pm 3.4 \%$ of visits in 2005 to 2007 and 2008 to 2010 ( $P_{\text {trend }}=0.001$ ), as shown in Figure 1B. Anticoagulants were not administered at $72.1 \pm 2.6 \%, 68.0 \pm 3.7 \%$ and $65.5 \pm 4.4 \%$ of visits in 2002 to 2004,2005 to 2007 and 2008 to $2010\left(\mathrm{P}_{\text {trend }}=0.146\right)$, as shown in Figure 1C. Approximately $52.3 \pm 2.8 \%, 52.0 \pm 4.1 \%$ and $47.2 \pm 4.9 \%$ of visits did not receive any antiplatelet agent (acetylsalicylic acid or thienopyridine/ GPI) in the ED during 2002 to 2004, 2005 to 2007 and 2008 to 2010, with no change over the study period $\left(\mathrm{P}_{\text {trend }}=0.352\right.$, Figure 1D).
In the subgroup of ED visits with a definitive primary diagnosis of AMI, acetylsalicylic acid was not administered at $48.7 \pm 4.5 \%, 44.7 \pm 6.2 \%$ and 55.5 $\pm 5.6 \%$ of visits in 2002 to 2004, 2005 to 2007 and 2008 to 2010. No significant change was noted during the study period $\left(P_{\text {trend }}=0.308\right)$, as shown in Figure 2A. The nonuse of thienopyridine/GPI in the ED at definitive AMI visits decreased from $81.9 \pm 3.4 \%$ in 2002 to 2004 to $77.0 \pm 5.0 \%$ in 2005 to 2007 and $71.1 \pm 5.5 \%$ in 2008 to 2010 , as shown in Figure $2 \mathrm{~B}\left(\mathrm{P}_{\text {trend }}=0.001\right)$. The nonuse of anticoagulants remained stable from $49.3 \pm 4.6 \%$ to $47.1 \pm 7.1 \%$ and $45.3 \pm 6.2 \%\left(P_{\text {trend }}=0.238\right)$ during the study period (Figure $2 \mathrm{C}$ ). In addition, the proportion of definitive AMI visits that did not receive any antiplatelet agent in the $\mathrm{ED}$ was $42.6 \pm 4.5 \%, 34.7 \pm 6 \%$ and $38.2 \pm 5.4 \%$ in 2002 to 2004,2005 to 2007 and 2008 to 2010, respectively ( $\left.P_{\text {trend }}=0.56\right)$ (Figure 2D).

The majority of AMI visits were not brought by an ambulance or emergency medical personnel, and the proportion did not change over the study period (Table 1). Acetylsalicylic acid was not administered at $47.7 \pm 4.7 \%, 42.0 \pm 6.0 \%$ and $45.6 \pm 7.3 \%$ of visits in 2002 to 2004 , 2005 to 2007 and 2008 to 2010 in this subpopulation, as shown in Figure 3A $\left(\mathrm{P}_{\text {trend }}=0.78\right)$. Similarly, $43.9 \pm 4.4 \%, 40.6 \pm 6.0 \%$ and $37.8 \pm 7.0 \%$ of visits for AMI not brought by ambulance did not receive any antiplatelet agent in 2002 to 2004, 2005 to 2007 and 2008 to 2010, as shown in Figure 3B $\left(\mathrm{P}_{\text {trend }}=0.42\right)$.

Predictors of antiplatelet and anticoagulant use in the emergency department

In the univariate models, acetylsalicylic acid use was associated with younger age, higher systolic blood pressure, arrival mode other than ambulance, presence of typical symptoms and ED location in an MSA 
TABLE 1

Characteristics of acute myocardial infarction (AMI) visits during the study period

\begin{tabular}{|c|c|c|c|c|}
\hline Variable & 2002-2004 & 2005-2007 & 2008-2010 & $\mathbf{P}_{\text {trend }}$ \\
\hline Number of visits, mean \pm SE & $1,999,941 \pm 152,343$ & $1,584,085 \pm 134,566$ & $1,441,038 \pm 156,406$ & NA \\
\hline Number of visits after exclusion, mean $\pm \mathrm{SE}$ & $1,602,774 \pm 146,975$ & $1,099,054 \pm 120,222$ & $1,124,077 \pm 137,744$ & NA \\
\hline Age, mean \pm SE & $64.30 \pm 0.96$ & $66.05 \pm 1.19$ & $64.01 \pm 1.18$ & $<0.001$ \\
\hline Length of visit, mean \pm SE & $301.60 \pm 23.05$ & $296.33 \pm 25.31$ & $269.11 \pm 18.89$ & $<0.001$ \\
\hline Systolic blood pressure, mean \pm SE & $140.90 \pm 1.82$ & $141.73 \pm 2.34$ & $146.22 \pm 2.95$ & $<0.001$ \\
\hline Heart rate, mean \pm SE & $87.61 \pm 1.42$ & $83.66 \pm 1.59$ & $85.08 \pm 1.79$ & $<0.001$ \\
\hline Female sex & $49.8 \pm 3.1$ & $45.3 \pm 3.4$ & $44.8 \pm 4.2$ & 0.309 \\
\hline \multicolumn{5}{|l|}{ Race } \\
\hline White & $83.1 \pm 2.5$ & $85.2 \pm 2.1$ & $79.8 \pm 3.3$ & 0.71 \\
\hline Black & $15.5 \pm 2.8$ & $14.0 \pm 2.5$ & $13.1 \pm 2.5$ & 0.523 \\
\hline Definitive diagnosis & $28.5 \pm 3.0$ & $33.4 \pm 4.4$ & $45.8 \pm 4.2$ & 0.001 \\
\hline Seen by ED attending physician & $95.7 \pm 1.1$ & $94.6 \pm 1.6$ & $95.8 \pm 2.0$ & 0.992 \\
\hline Seen by a consultant & $23.5 \pm 3.0$ & $15.5 \pm 2.5$ & $33.6 \pm 4.9$ & 0.104 \\
\hline Arrival by ambulance & $43.0 \pm 3.4$ & $46.5 \pm 4.6$ & $44.4 \pm 4.7$ & 0.814 \\
\hline Typical symptoms & $61.9 \pm 2.8$ & $59.6 \pm 3.7$ & $60.8 \pm 3.5$ & 0.786 \\
\hline Seen in ED of MSA & $85.0 \pm 3.5$ & $87.4 \pm 4.6$ & $88.7 \pm 4.2$ & 0.352 \\
\hline \multicolumn{5}{|l|}{ Geographical region of ED, United States } \\
\hline Northeast & $27.1 \pm 4.0$ & $28.3 \pm 4.2$ & $21.5 \pm 4.1$ & 0.391 \\
\hline Midwest & $22.2 \pm 2.8$ & $25.5 \pm 4.2$ & $22.2 \pm 4.1$ & 0.940 \\
\hline South & $29.8 \pm 3.9$ & $31.2 \pm 5.0$ & $34.4 \pm 5.7$ & 0.394 \\
\hline West & $20.9 \pm 3.0$ & $15.0 \pm 3.6$ & $21.9 \pm 4.9$ & 0.379 \\
\hline
\end{tabular}

Data presented as \% \pm SE unless otherwise indicated. ED Emergency department; MSA Metropolitan statistical area; NA Not applicable

TABLE 2

Unadjusted and adjusted ORs of receiving antiplatelet and anticoagulant therapies in the emergency department for acute myocardial infarction visits

\begin{tabular}{|c|c|c|c|c|c|c|}
\hline \multirow[b]{2}{*}{ Predictor variable } & \multicolumn{2}{|c|}{ Use of acetylsalicylic acid } & \multicolumn{2}{|c|}{ Use of thienopyridine or GPI } & \multicolumn{2}{|c|}{ Use of anticoagulant } \\
\hline & Unadjusted & Adjusted & Unadjusted & Adjusted & Unadjusted & Adjusted \\
\hline Age (per 10 years) & $0.81^{*}(0.73-0.90)$ & $0.89(0.78-1.01)$ & $0.86^{*}(0.75-0.97)$ & $0.95(0.82-1.10)$ & $0.94(0.85-1.03)$ & \\
\hline Female sex & $0.86(0.64-1.15)$ & & $0.51^{\star}(0.32-0.81)$ & $0.73(0.42-1.26)$ & $0.62^{*}(0.45-0.86)$ & $0.73(0.51-1.05)$ \\
\hline White & $0.86(0.63-1.22)$ & & $0.61(0.29-1.25)$ & & $0.71(0.45-1.13)$ & \\
\hline Black & $1.15(0.78-1.69)$ & & $1.22(0.65-2.28)$ & & $1.16(0.73-1.84)$ & \\
\hline Seen by ED physician & $1.78(0.75-4.22)$ & & $0.80(0.33-1.97)$ & & $1.72(0.68-4.34)$ & \\
\hline Length of visit (per $10 \mathrm{~min}$ ) & $1.00(0.99-1.01)$ & & $0.97^{\star}(0.95-0.98)$ & $0.97^{\dagger}(0.95-0.98)$ & $0.99(0.98-1.00)$ & \\
\hline SBP (per 10 mmHg) & $1.08^{*}(1.03-1.14)$ & $1.08^{\dagger}(1.02-1.15)$ & $0.99(0.93-1.07)$ & & $1.01(0.96-1.06)$ & \\
\hline Pulse (per 10 beats/min) & $0.99(0.98-1.00)$ & & $1.00(0.91-1.08)$ & & $1.02(0.94-1.10)$ & \\
\hline Seen by a consultant & $0.99(0.68-1.44)$ & & $2.40^{\star}(1.41-4.05)$ & $2.24^{\dagger}(1.22-4.13)$ & $1.56^{\star}(1.09-2.22)$ & $1.73^{\dagger}(1.14-2.63)$ \\
\hline Arrival by ambulance & $0.38^{*}(0.26-0.56)$ & $0.42^{\dagger}(0.28-0.62)$ & $1.37(0.90-2.11)$ & & $1.54^{*}(1.08-2.22)$ & $1.58^{\dagger}(1.11-2.25)$ \\
\hline Typical symptoms & $1.67^{*}(1.21-2.31)$ & $1.39(0.95-2.03)$ & $2.32^{*}(1.40-3.83)$ & $1.64(0.92-2.90)$ & $1.74^{*}(1.25-2.42)$ & $1.75^{\dagger}(1.20-2.55)$ \\
\hline ED located in MSA & $1.79^{*}(0.92-3.49)$ & $2.27^{\dagger}(1.02-5.09)$ & $3.30^{*}(0.93-11.75)$ & $3.08(0.81-11.71)$ & $2.39^{\star}(1.21-4.72)$ & $3.34^{\dagger}(1.54-7.24)$ \\
\hline ED located in Northeast & $1.25(0.85-1.82)$ & & $0.91(0.48-1.72)$ & & $0.79(0.50-1.23)$ & \\
\hline ED located in Midwest & $1.20(0.81-1.78)$ & & $0.72(0.31-1.67)$ & & $1.05(0.67-1.64)$ & \\
\hline ED located in South & $0.76(0.52-1.11)$ & & $1.50(0.85-2.66)$ & & $1.19(0.77-1.86)$ & \\
\hline ED located in West & $0.90(0.56-1.46)$ & & $0.88(0.48-1.62)$ & & $0.98(0.60-1.63)$ & \\
\hline
\end{tabular}

${ }^{\star} P<0.10 ;{ }^{\dagger}$ Bonferroni-adjusted P<0.05. Data presented as OR (95\% CI). ED Emergency department; GPI Glycoprotein IIB/IIIA; MSA Metropolitan statistical area; SBP Systolic blood pressure

(all $\mathrm{P}<0.10$ ). After adjustment for other predictors in a multivariable model, higher systolic blood pressure, arrival mode other than ambulance and ED location in an MSA remained associated with acetylsalicylic acid use (all Bonferroni-adjusted $\mathrm{P}<0.05$ ), as shown in Table 2.

Thienopyridine/GPI use was associated with younger age, male sex, shorter length of visit, evaluation by a consultant, presence of typical symptoms and ED location in an MSA (all $\mathrm{P}<0.10)$. As shown in Table 2, in the adjusted multivariable model, evaluation by a consultant and length of ED visit were the only independent predictors of thienopyridine/GPI use in AMI visits (all Bonferroni-adjusted $\mathrm{P}<0.05$ ).

Male sex, presence of typical symptoms, arrival by an ambulance, evaluation by a consultant and ED location in an MSA were associated with anticoagulation use in the ED (all $\mathrm{P}<0.10$; Table 2). As shown in Table 2, in the adjusted multivariable model, presence of typical symptoms, arrival by an ambulance, evaluation by a consulting physician and ED location in an MSA remained associated with anticoagulation use in the ED (all Bonferroni-adjusted $\mathrm{P}<0.05$ ).

\section{DISCUSSION}

NHAMCS is the only nationally representative survey that collects data regarding medications use during ED visits. Its probability sampling provides a unique opportunity to estimate and assess trends in the use of medications in the ED. The present analysis demonstrates that nonuse of antiplatelets and anticoagulants, including 


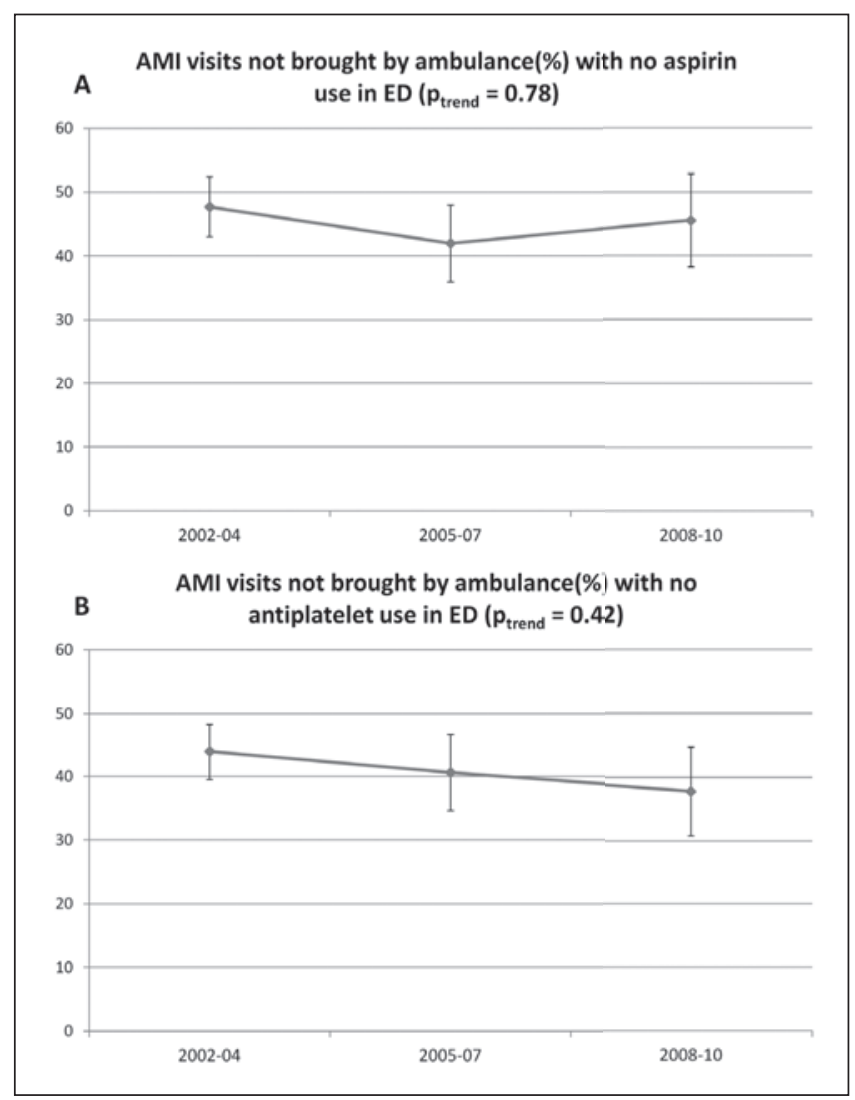

Figure 3) Proportion (\%) of acute myocardial infarction (AMI) visits not brought by ambulance which did not receive acetylsalicylic acid (aspirin)(A) or any antiplatelet agent in the emergency department (ED) (B). The vertical bar represents the standard error of the estimate

acetylsalicylic acid, for AMI visits in the ED is alarmingly high. Our results build on previous single-centre ED studies demonstrating acetylsalicylic acid use in only $30 \%$ and $56 \%$ of suspected AMI patients during 1994 and 1999 to 2002, respectively $(9,10)$. Furthermore, the present study extends the findings of previous NHAMCS studies reporting acetylsalicylic acid use in $34.3 \%$ and 40\% of AMI visits in the ED during 1995 to 1996 and 1998 to 2004, respectively $(11,12)$. We improved on previous studies by restricting our denominator to the extent possible in accordance with the AHA/ACC AMI performance measures statement (3).

We identified AMI ED visits based on any one of the three diagnosis entries on the patient record form, whether tentative or definitive. It is likely that some visits were due to non-AMI conditions, either associated with elevated enzymes or other findings raising suspicion of AMI. In the absence of contraindications, low-risk therapies, such as acetylsalicylic acid, may still be used in most cases before a definitive alternate diagnosis is reached. Nevertheless, subanalyses of the ED visits with a primary diagnosis of AMI that were not questionable, probable or rule out (ie, definitive AMI) was conducted. In this subgroup, not only is acetylsalicylic acid clearly indicated, but other therapies, such as thienopyridine/GPI and anticoagulants, should be used. Data regarding acetylsalicylic acid allergy or intolerance were not recorded in the NHAMCS. In these patients, alternate antiplatelet agents, such as thienopyridine, are indicated and typically used. To account for such visits, we estimated the proportion of AMI visits without antiplatelet use.

We suspected that visits in which the patient arrived by ambulance may have received acetylsalicylic acid en route; therefore, they were less likely to receive it in the ED (13) (Table 2). Consequently, a subanalysis of acetylsalicylic acid nonuse in visits not brought by ambulance was

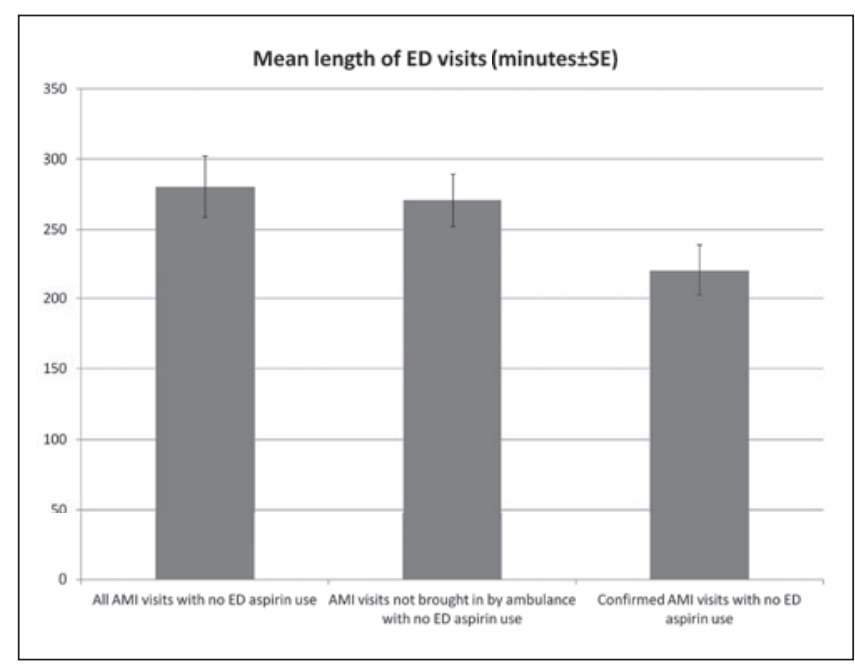

Figure 4) Mean length of visits in the emergency department (ED). The vertical bar represents the standard error of the estimate. AMI Acute myocardial infarction

conducted. However, even in this subgroup, acetylsalicylic acid nonuse remained disturbingly high (Figure 3). Our study found that nonuse of thienopyridine/GPI in the ED decreased over the study period for all AMI and definitive AMI visits. However, approximately $71 \%$ of the definitive AMI visits during 2008 to 2010 did not receive them in the ED. This finding is consistent with a recent report from the National Cardiovascular Data Registry that showed only $24.2 \%$ patients with non-ST elevation myocardial infarction received thienopyridine in the ED during 2007 to 2010 (14).

The Center for Medicare and Medicaid Services and the Joint Commission and ACC/AHA describe the administration of acetylsalicylic acid within $24 \mathrm{~h}$ of hospital arrival as a performance measure (1-3). However, AHA/ACC clinical practice guidelines for AMI recommend using acetylsalicylic acid as soon as possible (15-18). Although no clear randomized data support a time-sensitive effect of acetylsalicylic acid in the AMI, the International Study of Infarct Survival-2 (ISIS-2) showed a trend toward reduction in mortality with early use of acetylsalicylic acid alone (19). Furthermore, in ISIS-2, there was a higher reduction in mortality with early acetylsalicylic acid and streptokinase use ( $0 \mathrm{~h}$ to $4 \mathrm{~h}$ ) compared with treatment $5 \mathrm{~h}$ to $12 \mathrm{~h}$ and $13 \mathrm{~h}$ to $24 \mathrm{~h}$ after the symptom onset. Similarly, in the Argatroban in Acute Myocardial Infarction-2 study, early acetylsalicylic acid use $(1.6 \mathrm{~h}$ versus $3.5 \mathrm{~h})$ was associated with significant reduction in mortality at seven days, 30 days and one year of follow-up (20). In our study, the mean length of ED visits for all AMI visits, AMI visits not brought by an ambulance and the definitive AMI visits during which acetylsalicylic acid was not administered were $4.6 \mathrm{~h}, 4.5 \mathrm{~h}$ and $3.6 \mathrm{~h}$, respectively (Figure 4). Therefore, there may be a significant unrealized potential to reduce adverse events by administering acetylsalicylic acid on arrival based on the national estimate of untreated AMI ED visits. A similar gap exists with regard to thienopyridine and anticoagulant use for definitive AMI visits.

The exploratory analysis of variables associated with using acetylsalicylic acid, thienopyridine/GPI and anticoagulation should be considered hypothesis generating for future studies. One of the key patterns observed was the higher use of these therapies in the ED in MSA compared with non-MSA (Table 2). Of note, a 2009 National Healthcare Disparities report by the Agency for Healthcare Research and Quality recognizes non-MSA population as a priority due to significantly higher AMI mortality (21). We also found that visits evaluated by a consulting physician were more likely to receive thienopyridine/GPI and anticoagulants in the ED. The physicians' specialties are not identifiable from NHAMCS public use data files, 
but they were conceivably cardiologists or internal medicine physicians. An analysis of National Ambulatory Medical Care Survey and NHAMCS non-ED outpatient visits from 1993 to 2003 found that care by a cardiologist significantly increased the odds of acetylsalicylic acid use for secondary and primary prevention compared with other providers (22). Similarly, an analysis of the CRUSADE (Can Rapid risk stratification of Unstable angina patients Suppress ADverse outcomes with Early implementation of the ACC/AHA guidelines) registry found that high-risk acute coronary syndrome patients cared for by a cardiologist were more likely to receive early medications and had a lower risk of adjusted in-hospital mortality (23). Therefore, the effect of early involvement of a cardiologist on the use of evidencebased therapies and improvement in AMI outcomes needs to be evaluated in the future studies.

\section{Limitations}

Our study had several limitations. The validity of the results depends on the accuracy of the data abstraction. There is a well-defined procedure for training of local hospital representatives and Census Bureau field representatives involved in the data abstraction (8), although the accuracy of data abstraction, especially reporting on medication use, has been questioned in the past based on divergent results of NHAMCS and local ED-based studies (24). However, the staff at CDC and NHAMCS cited the difference in study methodology and national representation of the NHAMCS sample as the primary reason for the divergent results, although small errors in data abstraction are possible (25). A National Emergency Department Safety Study analyzing data from 58 US EDs reported acetylsalicylic acid use in $83 \%$ of AMI patients; however, the included population was not nationally representative (26). All EDs included in the present study were located in urban areas, $78 \%$ were affiliated with an emergency medicine residency program and $91 \%$ were associated with cardiac catheterization laboratories. On the other hand, a report from the CRUSADE registry of high-risk patients with non-ST elevation acute coronary syndrome presenting to the ED from April 2003 to December 2004 found that approximately $30 \%$ of patients with no prehospital use of acetylsalicylic acid did not receive acetylsalicylic acid in the ED (27). This finding is similar to nonuse of acetylsalicylic acid in visits not brought by ambulance in our analysis (Figure 3) allowing differences in databases, ie, a voluntary quality improvement registry versus a nationally

\section{REFERENCES}

1. US Governement Accountability Office. Hospital Quality Data: CMS Needs More Rigorous Methods to Ensure Reliability of Publicly Released Data. Washington, DC: US Government Accountability Office; 2006. Publication GAO-06-54.

2. Williams SC, Schmaltz SP, Morton DJ, et al. Quality of care in US hospitals as reflected by standardized measures, 2002-2004. N Engl J Med 2005;21:255-64.

3. Krumholz HM, Anderson JL, Bachelder BL, et al. ACC/AHA 2008 performance measures for adults with ST-elevation and non-STelevation myocardial infarction: A report of the American College of Cardiology/American Heart Association Task Force on Performance Measures (writing committee to develop performance measures for ST-elevation and non-ST-elevation myocardial infarction) developed in collaboration with the American Academy of Family Physicians and American College of Emergency Physicians, endorsed by the American Association of Cardiovascular and Pulmonary Rehabilitation, Society for Cardiovascular Angiography and Interventions, and Society of Hospital Medicine. J Am Coll Cardiol 2008;52:2046-99.

4. Roe MT, Messenger JC, Weintraub WS, et al. Treatments, trends, and outcomes of acute myocardial infarction and percutaneous coronary intervention. J Am Coll Cardiol 2010;56:254-63.

5. Mehta RH1, Roe MT, Chen AY, et al. Recent trends in the care of patients with non-ST-segment elevation acute coronary syndromes: Insights from the CRUSADE initiative. Arch Intern Med 2006;166:2027-34.

6. George MG, Tong X, Sonnenfeld N, et al Centers for Disease Control and Prevention (CDC). Recommended use of aspirin and other antiplatelet medications among adults - National Ambulatory representative sample. Approximately $60 \%$ of patients with suspected cardiac symptoms may receive acetylsalicylic acid in the ambulance $(13,28)$. Therefore, a separate analysis for patients not brought in by ambulance was performed. However, $5 \%$ to $26 \%$ patients may selfadminister acetylsalicylic acid before the ED visit depending on previous use of acetylsalicylic acid (29). Inability to account for self-administration of acetylsalicylic acid before the ED visit may have led to overestimation of its nonuse in the ED. However, only $22.3 \%$ patients with cardiac history self-administered acetylsalicylic acid even after an educational intervention in a randomized controlled trial (30); therefore, self-administration probably did not have a major effect on our estimates.

We could not exclude patients with allergies or health care provider-documented contraindications from the denominator. We conducted analysis for any antiplatelet use, as guidelines recommend use of thienopyridine in patients allergic to acetylsalicylic acid. However, we may still have overestimated the nonuse of acetylsalicylic acid due to the inability to account for documented contraindications not captured by ICD-9 and reason for visit codes. Also, some visits may have been soft rule out, but even in patients with definitive AMI diagnosis, the nonuse of antiplatelet and anticoagulant remained unsatisfactory. We specifically used the ED diagnosis and not the discharge diagnosis because decisions in ED are based on the former. Finally, it is possible that in a small proportion of the ED visits acetylsalicylic acid was not used due to urgent transfer to a cardiac catheterization laboratory. However, as indicated previously, the majority of ED patients that did receive acetylsalicylic acid stayed in ED for $\geq 3$ h (Figure 4).

\section{CONCLUSION}

The nonuse of evidence-based antiplatelet and anticoagulant therapies in the ED for AMI remains alarmingly high. There is some improvement in using thienopyridine/GPI, but the unsatisfactory use of acetylsalicylic acid has not improved over the years. A quality improvement initiative directed at the delivery of these agents in the ED may result in improvement of AMI outcomes.

DISCLOSURES: The authors have no conflicts of interest related to this article.

Medical Care Survey and National Hospital Ambulatory Medical Care Survey, United States, 2005-2008. MMWR Morb Mortal Wkly Rep 2012;(61 Suppl):11-8.

7. McCaig LF, Burt CW. Understanding and interpreting the National Hospital Ambulatory Medical Care Survey: Key questions and answers. Ann Emerg Med 2012;60:716-21.

8. Saketkhou BB, Conte FJ, Noris M, et al. Emergency department use of aspirin in patients with possible acute myocardial infarction. Ann Intern Med 1997;127:126-9.

9. Takakuwa KM, Shofer FS, Hollander JE. Aspirin administration in ED patients who presented with undifferentiated chest pain: Age, race, and sex effects. Am J Emerg Med 2010;28:318-24.

10. Burt CW. Summary statistics for acute cardiac ischemia and chest pain visits to United States EDs, 1995-1996. Am J Emerg Med 1999;17:552-9.

11. Pham JC, Kelen GD, Pronovost PJ. National study on the quality of emergency department care in the treatment of acute myocardial infarction and pneumonia. Acad Emerg Med 2007;14:856-63.

12. McVaney KE, Macht M, Colwell CB, et al. Treatment of suspected cardiac ischemia with aspirin by paramedics in an urban emergency medical services system. Prehosp Emerg Care 2005;9:282-4.

13. Diercks DB, Kontos MC, Hollander JE, et al. ED administration of thienopyridines in non-ST-segment elevation myocardial infarction: Results from the NCDR. Am J Emerg Med 2013;31:1005-11.

14. Antman EM, Anbe DT, Armstrong PW, et al. ACC/AHA guidelines for the management of patients with ST-elevation myocardial infarction - executive summary. A report of the American College of Cardiology/American Heart Association Task Force on Practice Guidelines (writing committee to revise the 1999 guidelines for the 
management of patients with acute myocardial infarction). Circulation 2004;110:588-636.

15. Braunwald E, Antman EM, Beasley JW, et al. American College of Cardiology/American Heart Association Task Force on Practice Guidelines (committee on the management of patients with unstable angina). Circulation 2002;106:1893-900.

16. O'Gara PT, Kushner FG, Ascheim DD, et al. 2013 ACCF/AHA Guideline for the Management of ST-Elevation Myocardial Infarction: A Report of the American College of Cardiology Foundation/American Heart Association Task Force on Practice Guidelines. J Am Coll Cardiol 2013;61:78-e140.

17. Wright RS, Anderson JL, Adams CD, et al. 2011 ACCF/AHA Focused Update of the Guidelines for the Management of Patients With Unstable Angina/ Non-ST-Elevation Myocardial Infarction (Updating the 2007 Guideline): A report of the American College of Cardiology Foundation/American Heart Association Task Force on Practice Guidelines. Circulation 2011;123:2022-60.

18. ISIS-2. Second International Study of Infarct Survival Collaborative Group. Randomised trial of intravenous streptokinase, oral aspirin, both or neither among 17,187 cases of suspected acute myocardial infarction. Lancet 1988;2:349-60.

19. Freimark D, Matetzky S, Leor J, et al. Timing of aspirin administration as a determinant of survival of patients with acute myocardial infarction treated with thrombolysis. Am J Cardiol 2002;89:381-5. <www.ahrq.gov/research/findings/nhqrdr/nhdr09/ Chap4e.html> (Accessed January 5, 2014).

20. Stafford RS, Monti V, Ma J. Underutilization of aspirin persists in US ambulatory care for the secondary and primary prevention of cardiovascular disease. PLoS Med 2005;2:e353.
21. Roe MT, Chen AY, Mehta RH, et al. Influence of inpatient service specialty on care processes and outcomes for patients with non ST-segment elevation acute coronary syndromes. Circulation 2007;116:1153-61.

22. Cooper RJ. NHAMCS: Does it hold up to scrutiny? Ann Emerg Med 2012;60:722-5.

23. McCaig LF, Burt CW, Schappert SM, et al. NHAMCS: Does it hold up to scrutiny? Ann Emerg Med 2013;62:549-51.

24. Tsai CL, Magid DJ, Sullivan AF, et al. Quality of care for acute myocardial infarction in 58 U.S. emergency departments. Acad Emerg Med 2010;17:940-50.

25. Fermann GJ, Raja AS, Peterson ED, et al. Early treatment for non-ST-segment elevation acute coronary syndrome is associated with appropriate discharge care. Clin Cardiol 2009;32:519-25.

26. Colwell C, Mehler P, Harper J, et al. Measuring quality in the prehospital care of chest pain patients. Prehosp Emerg Care 2009;13:237-40.

27. Jaffy MB, Meischke H, Eisenberg MS. Prevalence of aspirin use among patients calling 9-1-1 for chest pain. Acad Emerg Med 1998;5:1146-9.

28. Dracup K, McKinley S, Riegel B, et al. A randomized clinical trial to reduce patient prehospital delay to treatment in acute coronary syndrome. Circ Cardiovasc Qual Outcomes 2009;2:524-32.

29. Jaffy MB, Meischke H, Eisenberg MS. Prevalence of aspirin use among patients calling 9-1-1 for chest pain. Acad Emerg Med 1998;5:1146-9.

30. Dracup K, McKinley S, Riegel B, et al. A randomized clinical trial to reduce patient prehospital delay to treatment in acute coronary syndrome. Circ Cardiovasc Qual Outcomes 2009;2:524-32. 